\title{
A stress free model for residual stress assessment using thermoelastic stress analysis
}

\author{
Geoffrey Howell ${ }^{1}$, Janice M. Dulieu-Barton, Mithila Achintha and Andrew F. Robinson \\ Faculty of Engineering and the Environment, University of Southampton, UK. \\ ${ }^{1}$ G.Howell@soton.ac.uk
}

\begin{abstract}
Thermoelastic Stress Analysis (TSA) has been proposed as a method of obtaining residual stresses. The results of a preliminary study demonstrated that when Al-2024 plate containing holes that were plastically deformed by cold expansion process to $2 \%$ and $4 \%$ strain the thermoelastic response in the material around the hole was different to that obtained from a plate that had not experienced any plastic cold expansion (i.e. a reference specimen). This observation provides an opportunity for obtaining residual stresses based on TSA data. In many applications a reference specimen (i.e. residual stress free specimen) may not be available for comparison, so a synthetic, digital bitmap has been proposed as an alternative. An elastic finite element model is created using commercially available software Abaqus/Standard and the resultant stress field is extracted. The simulated stress field from the model is mapped onto a grid that matches the TSA pixel data from a physical reference specimen. This stress field is then converted to a $\Delta T / T$ field that can be compared to the full-field TSA data. When the reference experimental data is subtracted from the, bitmap dataset the resultant $\Delta T / T$ field is approximately zero. Further work proposes replacing the experimental reference data with that from specimens that have undergone cold expansion with the aim of revealing the regions affected by residual stress through a departure from zero in the resultant stress field. The paper demonstrates the first steps necessary for deriving the residual stresses from a general specimen using TSA.
\end{abstract}

Keywords: finite element, plastic strain, residual stress, Thermoelastic Stress Analysis, TSA.

\section{INTRODUCTION}

Thermoelastic Stress Analysis (TSA) ${ }^{[1]}$ is a non-destructive, full-field stress analysis technique which is based on the measurement of small temperature changes resulting from elastic loading. For linear, isotropic, homogenous materials the temperature change is directly proportional to the change in the sum of the principal surface stresses ${ }^{[1]}$. The usual practice is to measure the change in temperature with the aid of a highly sensitive IR detector. Residual stresses can be created during manufacturing, assembly, surface treatments, or during service life. Knowing the stress state within a component is important as premature failure of the component can be caused through the combination of residual stress and in-service stresses. There are currently several methods for measuring the residual stress within a component such as hole drilling ${ }^{[2]}$, contour method ${ }^{[3]}$, and X-ray diffraction ${ }^{[4]}$; however they are either destructive, time consuming, expensive, only acquire point-data rather than full-field data, or require the component to be removed from service for a considerable length of time and examined in a laboratory. Therefore an opportunity arises for a relatively cheap, quick, robust, full-field measurement system that allows the non-destructive derivation of the residual stress in a component, without the need to remove the component from service. TSA has previously been proposed as a solution; previous work ${ }^{[5]}$ has shown that the differences in thermoelastic response due to a material experiencing different levels of plastic deformation can be detected using TSA. Through comparison of the thermoelastic response of a component that has experienced plastic straining to the response of a reference component that has not experienced plastic straining, regions experiencing plastic straining can be identified. In an industrial application, it is very unlikely that there will be a reference specimen available to allow this comparison to be performed. It is proposed that a hybrid method of using a numerically generated reference specimen as an alternative to a physical reference specimen could yield comparable results when investigating changes in $\Delta T / T$; an assessment of the feasibility of using a simulated bitmap in place of a reference specimen to identify changes in $\Delta T / T$ is being presented here. A further calibration would then be required to convert the $\Delta T / T$ within these regions into values of plastic strain. It is then envisaged that the plastic strain values would be used in conjunction with a non-linear finite element model, from which the amount of residual stress resulting from the plastic strain can be extracted thereby creating a new hybrid techniques for obtaining residual stresses. 


\section{CURRENT RESIDUAL STRESS ASSESSMENT TECHNIQUES}

Residual stress assessment techniques can be described as either destructive or non-destructive; the proposed technique of using a hybrid approach based on TSA falls into the latter category. Of the destructive techniques described, they are all often unsuitable for use on components that are to be returned to service as they usually result in the removal of material required for structural integrity; although this is not always the case, large structures can have holes drilled and filled afterwards with little detrimental effect ${ }^{[6]}$. The destructive techniques also can only inform the user of the stress field close to the point of material removal, so may require several areas of a structure to be tested to build a full map of the stress state within a specimen. These techniques can provide an analysis of the through thickness stress distribution if incremental material removal is performed, although in the case of the contour method the stress field is only the field normal to the cut direction through the material.

Non-destructive techniques offer a way of measuring the residual stresses near the surface of a component, and also allow the component to be returned to service after the analysis has taken place. However, these techniques can be very expensive and often require the components to be taken to specific laboratories for the tests to take place. Synchrotron and neutron diffraction techniques require large and expensive radiation sources, and although they can penetrate to a depth of up to $150 \mu \mathrm{m}$ they are not suitable for long term maintenance monitoring schedules as the equipment is not readily available. X-ray diffraction is more readily available but it still requires the components to be removed from service and taken to a laboratory for testing. Ultrasound assessment ${ }^{[7]}$ is a new technique that can be performed in the field, however it cannot easily differentiate the effects of multi-axial stresses and requires a high degree of skill to be utilised effectively.

With these limitations on traditional destructive and non-destructive techniques, TSA would provide another nondestructive approach that could be used on components in the field without the need to remove them from service. It is a relatively cheap approach, which does not require the use of high energy radiation sources. It provides full-field data but must have a line of sight to the component under investigation.

\section{THERMOELASTIC STRESS ANALYSIS}

Thermoelastic Stress Analysis (TSA) ${ }^{[1]}$ is an experimental technique which utilises infra-red thermography. TSA is based upon the small changes in temperature that arise due to a material being subjected to a change in elastic strain; this is generally referred to as the thermoelastic effect. When a specimen is placed under cyclic loading conditions, the change in strain creates a cyclic variation in temperature. This temperature change $(\Delta T)$ (known as the thermoelastic response) can be related to the change in the first stress invariant $\left(\Delta\left(\sigma_{1}+\sigma_{2}\right)\right)$, i.e. the sum of the principal stresses ${ }^{[1]}$. An infra-red (IR) detector array can be used to measure the change in temperature, and from this the stresses can be calculated using the following relationship:

$$
\Delta T=-K T \Delta\left(\sigma_{1}+\sigma_{2}\right)
$$

where $T$ is surface temperature, and $K$ is the thermoelastic constant. $K=\alpha / \rho C_{p}$ and $\alpha, \rho, C_{p}$ are the coefficient of linear thermal expansion, density, and specific heat at constant pressure respectively.

Equation (1) is valid for linear elastic, homogenous materials where the temperature change has taken place under adiabatic conditions ${ }^{[1]}$. Equation (1) cannot be used for residual stress assessment; the stress term is the change in the principal stresses so it independent of any residual stresses contained in the component and the mean stress resulting from the applied cyclic load. Most importantly in the context of the present work, for equation 1 to be valid, a further assumption is made that the elastic mechanical properties are not temperature dependent.

It was Belgen ${ }^{[8]}$ who first observed that equation (1) is not always valid and proposed that the mean stress may affect the temperature change. In 1988 , Wong et al ${ }^{[9]}$ proposed the general theory be revised such that it included the temperature dependence of the elastic properties as follows:

$$
\dot{T}=\frac{T}{\rho C_{p}}\left[-\left(\alpha+\left(\frac{v}{E^{2}} \frac{\partial E}{\partial T}-\frac{1}{E} \frac{\partial v}{\partial T}\right) \sigma_{k k}\right) \dot{\sigma}_{k k}+\left(\frac{(1+v)}{E^{2}} \frac{\partial E}{\partial T}-\frac{1}{E} \frac{\partial v}{\partial T}\right) \sigma_{i j} \dot{\sigma}_{i j}\right]
$$


where $\dot{T}$ is the rate of change of temperature, $E$ is Young's modulus, $v$ is Poisson's ratio, $\sigma_{k k}$ is the trace of the stress tensor, and $\sigma_{i j}$ is the stress tensor. This new equation is known as the revised higher order thermoelastic theory and includes the temperature dependence of the elastic properties.

To simplify equation (2) only uniaxial loading will be considered (where $\sigma_{k k}=\sigma_{11}$, and $\sigma_{22}=\sigma_{12}=0$ ), to give:

$$
\dot{T}=-\frac{T}{\rho C_{p}}\left(\alpha-\frac{1}{E^{2}} \frac{\partial E}{\partial T} \sigma_{11}\right) \dot{\sigma}_{11}
$$

Equation (3) shows that the rate of temperature change is a function of both the applied stress, and its rate of change. In TSA $\sigma_{11}$ could be considered the mean stress $\sigma_{m}$ (which is the combination of residual stress in the material and the mean of the applied stress range during TSA), and so integrating equation (3) over the period of the loading provides a relationship between change in temperature and the change in stress:

$$
\Delta T=-\frac{T}{\rho C_{p}}\left(\alpha-\frac{1}{E^{2}} \frac{\partial E}{\partial T} \sigma_{m}\right) \Delta \sigma_{11}
$$

The revised higher order theory equation enables mean stress dependence of the thermoelastic constant to be accounted. Thus, the thermoelastic response is dependent on the mean stress as well as the applied stress, as shown in equation (4). Wong et $a l^{[10]}$ performed a validation of equation (4) using two uniaxially loaded aluminium specimens. One specimen was manufactured as an undeformed bar, the other was manufactured bent and straightened using a 4-point load until it was geometrically similar. Strain gauges were used to confirm areas of tensile and compressive strain in the straightened specimen. Results from the TSA of both specimens showed significant differences between the two specimens; this was attributed to residual stress in the straightened specimens. There was good agreement between the strain gauge readings and values given by equation (4). Dunn et $a l^{[11]}$ have confirmed that the mean stress effect is measurable for three materials; titanium (Ti-6Al-4V), aluminium (Al-2024), and steel (4340). There was an observable mean stress dependence of the thermoelastic constant, with $\frac{\partial K}{\partial \sigma_{m}} \cdot \frac{1}{K}=0.45,0.31$, and $0.11 \mathrm{GPa}^{-1}$ respectively.

It was shown ${ }^{[12]}$ that when plastic deformation is introduced, the thermoelastic constant is modified in some metals. From this, it was suggested that the change in thermoelastic constant could be used to estimate the level of plastic strain experienced by a component. Plastic straining is one of the causes of residual stress, so it may be possible that the residual stress within a component could be derived using TSA. In materials where the temperature dependence of the Young's modulus is small the modification in the thermoelastic constant could be used to derive the residual stresses instead of obtaining the mean stresses as in equation (2). Such a material is $316 \mathrm{~L}$ stainless steel used extensively in the nuclear power industry. The first step is to identify the regions of where the thermoelastic constant has been modified and for this a residual stress free reference data is required that represents the thermoelastic response. Once the regions containing residual stress have been identified then a calibration procedure would be used to determine the modification in the thermoelastic constant and hence the amount of plastic straining experienced. The plastic strain would then be used in conjunction with nonlinear FEA to provide an assessment of the residual stresses.

\section{EXPERIMENTAL WORK}

In the present paper previously collected and validated experimental data is used. A full description of the experimental work can be found in Robinson et $a l^{[13]}$. Essentially aluminium alloy plates with holes that had been cold expanded holes were produced to investigate the change in thermoelastic response due to the residual stresses and the plastic straining experienced in the neighbourhood of the hole. The plates were made from cold rolled aluminium alloy 2024 and were $150 \mathrm{~mm}$ wide x 300 long x $10 \mathrm{~mm}$ thick. Central circular holes of diameter $16 \mathrm{~mm}$ were drilled as shown in Figure 1 . The area around the centre section of the plates were coated in a layer of RS matt black spray paint before being mounted in a $100 \mathrm{kN}$ Instron 8800 servo-hydraulic test machine. The plates were cyclically loaded at $30 \pm 25 \mathrm{kN}(20 \pm 16.7 \mathrm{MPa})$. The stress range was selected to keep the maximum stress at the holes below the yield stress $(\sim 180 \mathrm{MPa})$. The thermoelastic response was measured using a Cedip Silver 480M IR detector. The viewing window of the detector was an $84 \times 62 \mathrm{~mm}$ rectangle centred on the holes in the plates.

Alongside the specimens that contained the cold expanded holes a reference specimen was made which contained a hole that had not undergone any cold expansion. In ${ }^{[13]}$ the TSA data from the plates with the cold expanded hole compared to the results from the reference specimen. The reference data set was subtracted from the data sets with the cold expanded 
holes. This procedure revealed a clear region in the neighbourhood of the holes where there was a difference in the response. It was shown ${ }^{[13]}$ that this region corresponded to the region of compressive residual stresses produced by the cold expansion process. Here it should be noted that the change in response is due to mainly the temperature dependence of Young's modulus and not the modification of the thermoelastic constant. The residual stresses were measured using $\mathrm{X}$-ray diffraction and incorporated into Equation (2) along with all the relevant material properties. It was shown that the thermoelastic response calculated from equation (2) corresponded almost exactly to the measured thermoelastic response.

For a general industrially relevant component it will be at best costly and difficult to produce a reference specimen. So the purpose of the present paper is to demonstrate that this can be done by producing a synthetic bitmap of TSA data using a linear elastic finite element analysis. Therefore the reference specimen used in ${ }^{[13]}$ will be used as a validation tool for the modelling approach.

The Altair LI software used in the Cedip IR system produces data sets of $T$, the surface temperature reading and $\Delta T$, the thermoelastic response. This means that a normalised thermoelastic response $\Delta T / T$ can be obtained that accounts for any differences in the specimen surface temperature during the test. These two datasets for the reference specimen were imported into MatLab and manipulated to obtain a $\Delta T / T$ dataset as shown in Figure 2.

To obtain the thermoelastic constant, $K$, given in equation (1) it is necessary to carry out an experiment on a specimen made from the same material as the component and apply a known stress. In this case it was decided to load a small dogbone specimen in uniaxial tension, hence $\sigma_{2}$ in equation (1) is zero and $\sigma_{1}$ can be determined from the range of the applied cyclic load and the cross sectional area of the specimen. The specimen was cyclically loaded at $4 \pm 3 \mathrm{kN}$, so the thermoelastic constant, $K$, was derived as $9.85 \times 10^{-12} \mathrm{~Pa}^{-1}$. The $K$ value can be used with the experimentally derived $\Delta T / T$ values to obtain the stress sum or with the stress sum values derived from the FEA to provide $\Delta T / T$. Here the latter approach is used to provide a synthetic thermoelastic bitmap. 


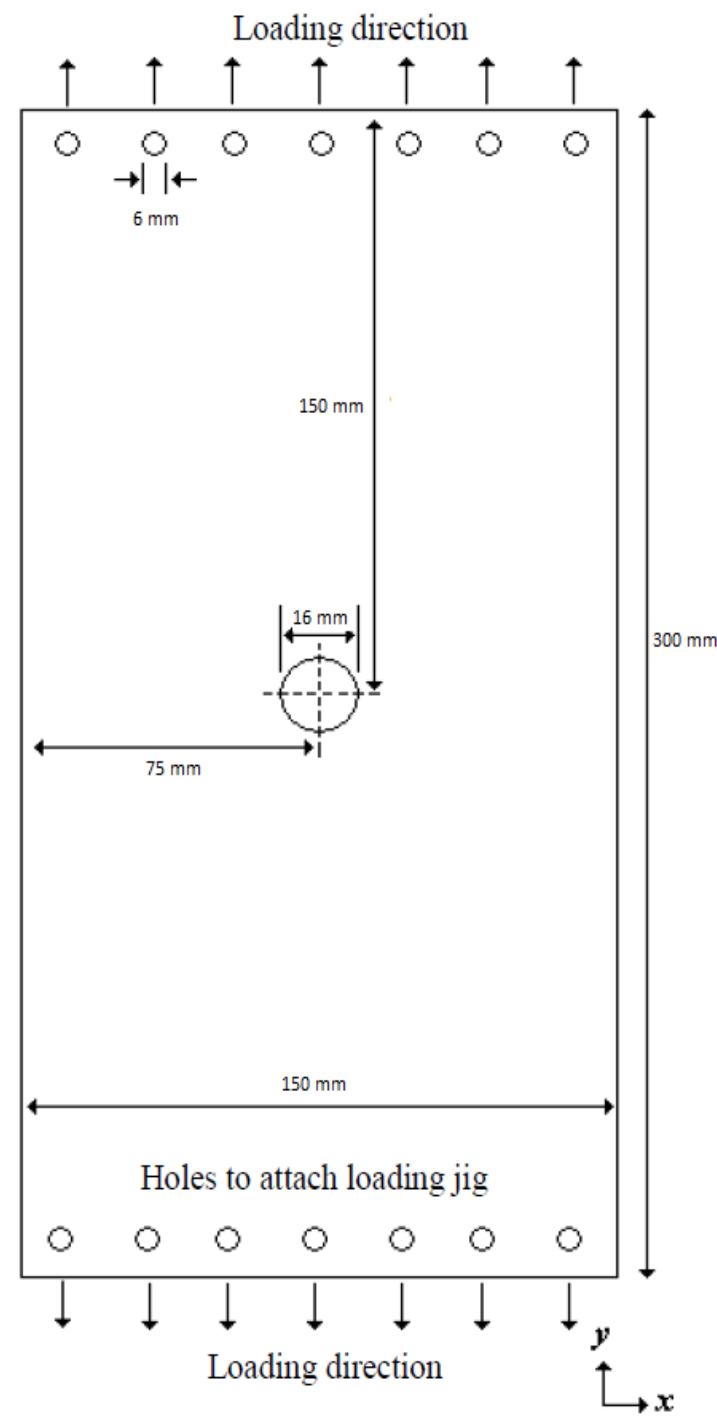

Figure 1 - Dimensions of the plate-with-hole specimens ${ }^{[13]}$.

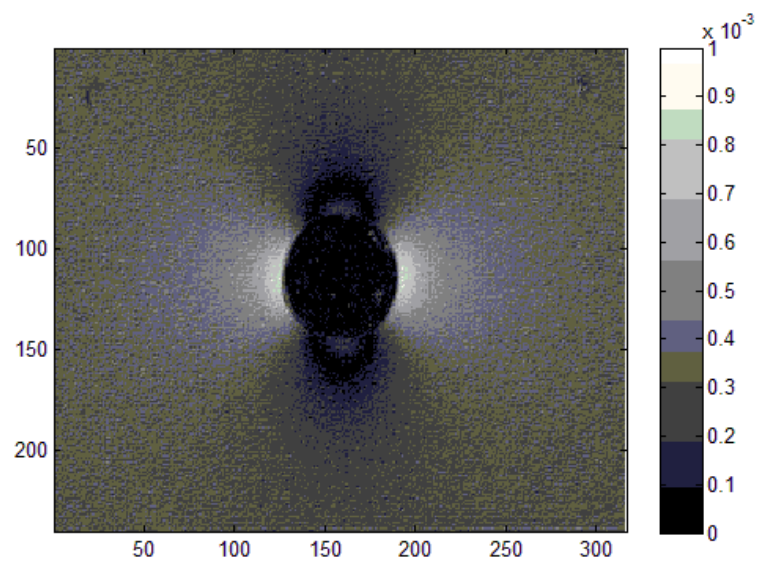

Figure 2 - Experimentally derived $\Delta \mathrm{T} / \mathrm{T}$ data around the hole. 


\section{DEVELOPMENT AND USE OF A SYNTHETIC BITMAP}

To develop a model that can be matched to the full-field data provided by TSA, a detailed FEA model needs to be constructed with elements that are at a similar resolution to the experimental data. Therefore it was decided that a submodelling approach was to be used, to enable the same region of interest used in the experimental work to be modelled. A 2D plane stress global model, of the plate, was produced in Abaqus/Standard (Abaqus 6.13-3). The model had the same dimensions as the plate given in Figure 2 and is shown in Figure 4. Elastic material properties of $E=$ $74.8 \mathrm{GPa}$ and $v=0.3^{[13]}$, and an average element size of $5 \mathrm{~mm}$ was used in the global model to achieve low run times with an acceptable degree of accuracy. A zero longitudinal displacement constraint was applied to one end of the specimen and a uniaxial, tensile pressure of $33.3 \mathrm{MPa}$ (load range/cross sectional area $\left[50 \mathrm{kN} / 1500 \mathrm{~mm}^{2}\right]$ ) applied at the other. Within the model database a second 2D plane stress model was made that measured $84 \times 62 \mathrm{~mm}$ and contained a hole of diameter $16 \mathrm{~mm}$ at its centre. This model was meshed with an average element size of $0.3 \mathrm{~mm}^{2}$, this value was found through a brief mesh density study that focused on maximum stress along the $\mathrm{x}$ axis through the centre of the plate. Once the change in maximum stress was found to be less than $3 \%$ between simulations, and the asymptote the values were approaching was $~ 95 \mathrm{MPa}$ ([applied stress] $\mathrm{x}$ [stress concentration factor]), it was decided that the mesh was refined enough for the purposes of this feasibility study. Both plate models had their origins at the centre of the hole, so the border of the new plate matched the partition put into the larger, original model. This allowed submodel boundary conditions to be applied, which in Abaqus is the application of the nodal displacements from the nodes closest to the selected area in the global model. Due to the inclusion of the partition there were nodes on the boundary already, however this would not be a requirement for further tests as a linear interpolation is applied by Abaqus if there are not any suitable nodes.

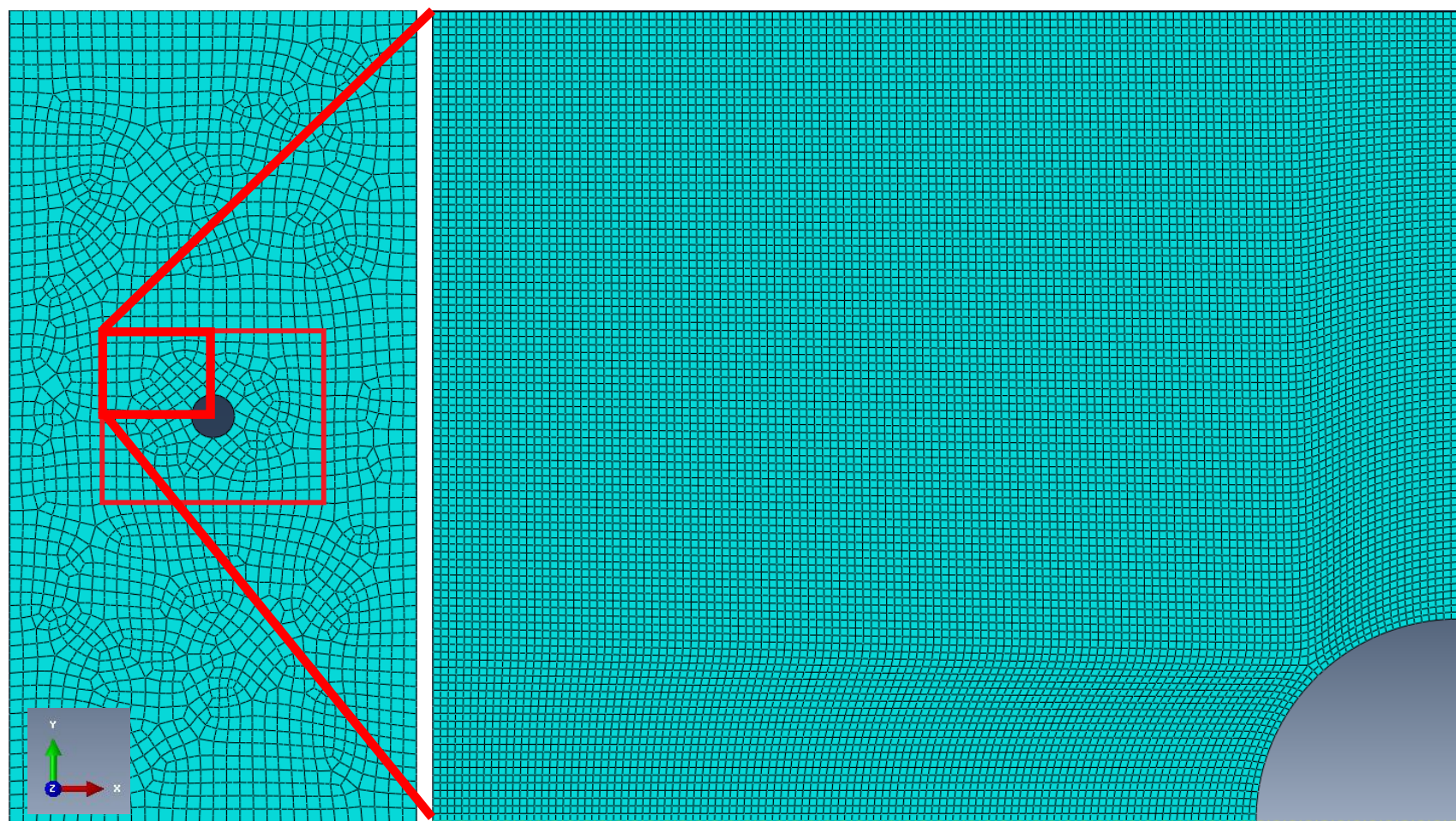

Figure 3 - Abaqus model of the hole-in-plate specimen with partition for submodel around the hole ( $300 \times 150 \mathrm{~mm}$ plate with a $16 \mathrm{~mm}$ diameter hole, and a $84 \times 62 \mathrm{~mm}$ partition, element size $5 \mathrm{~mm}$ ), (right) $1 / 4$ of the submodel (element size $0.3 \mathrm{~mm}$ ) with displacement boundary conditions taken from the nodes in the global model and applied on the outside edge of the submodel.

These elastic models were run and the $\sigma_{x}$ and $\sigma_{y}$ outputs from the model were exported into MatLab. The sum of the principal stress is an invariant and hence $\left(\sigma_{x}+\sigma_{y}\right)=\left(\sigma_{1}+\sigma_{2}\right)$, so the coordinate stresses at each node were simply added. The data set was smaller and less dense than that of the TSA, and it was not in a linear grid. The data was transformed into a grid of $255 \times 320$ elements using the $\mathrm{x}$-y coordinate data from Abaqus, and any additional data to fill the data set was created through a linear interpolation run through MatLab 2013a (the interpolation function is not 
available in previous versions of MatLab). The FE dataset contained both negative and positive values, whilst the experimental datasets (Figure 2) contain only the magnitude of $\Delta T / T$, so the absolute values of the FEA data were taken and can be seen in Figure 4. The experimentally determined value of $K$ for the aluminium was used to convert the $\Delta\left(\sigma_{x}+\sigma_{y}\right)$ datasets into $\Delta T / T$ synthetic bitmap, from which the experimental bitmap was subtracted to give the plot shown in Figure 5. In this final dataset it can be seen that there is very little difference between the FEA dataset and the TSA dataset for $\Delta T / T$. Visible in the image is the noise from the experimental data, and from Figure 6 it can be seen that the noise is an order of magnitude less than the $\Delta T / T$ results from the either the experiment or the simulation. The uniform distribution of the noise indicates there is not any bias in the image. At the top and bottom of the hole in the centre of the image there is a slight aberration, the origin of which comes from a combination of slight motion from the captured TSA data, the filling of the synthetic bitmap by the interpolation routine, and the effects due to the free edge around the hole.

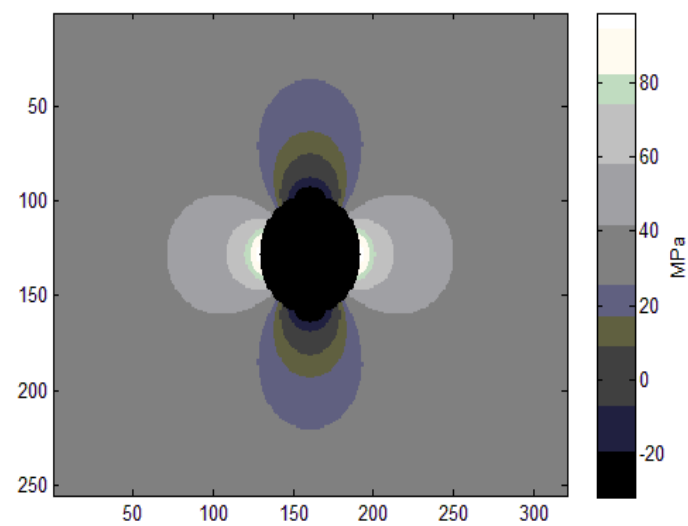

Figure 4 - Plot of the stress found in the FEA model.

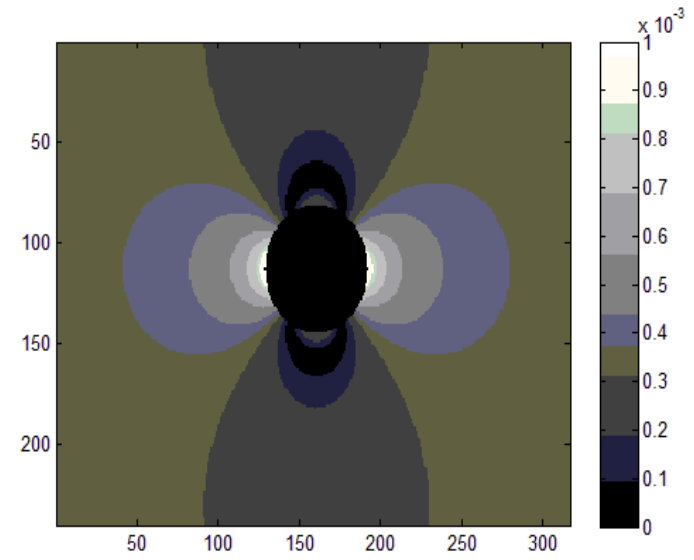

Figure 5 - Simulated thermoelastic bit map.

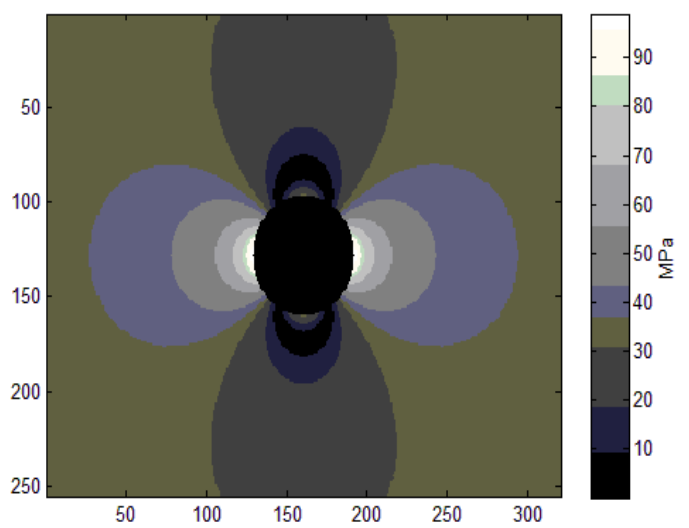

Plot of the magnitude of the stress.

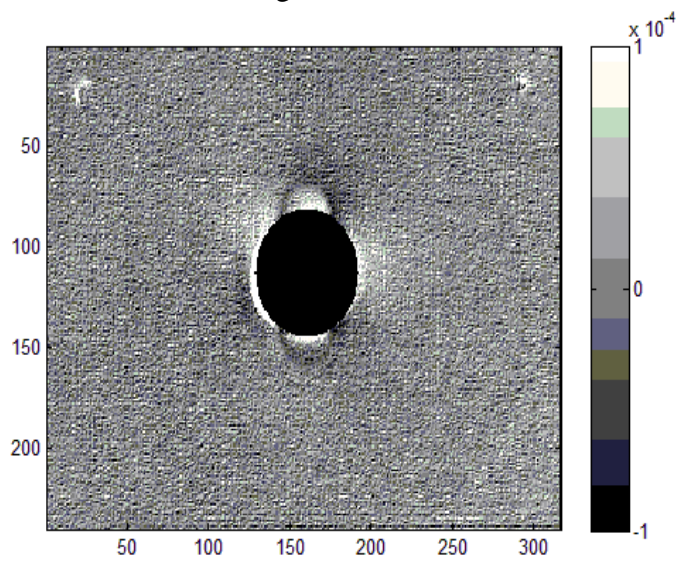

Plot of the simulated bit map - experimental bit map. 


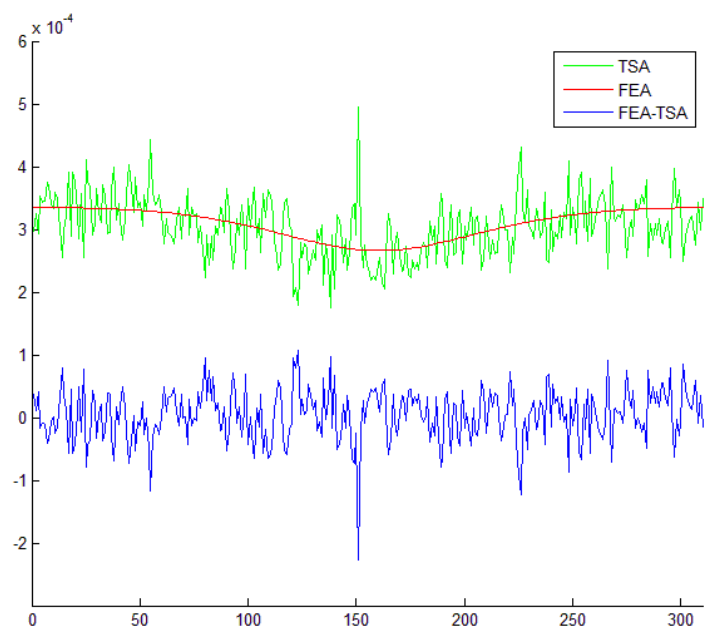

Figure 6 - Line plots showing the $\Delta T / T$ in the far field for the simulated (FEA) and the experimental (TSA) bit maps. The subtracted $\Delta T / T$ result is also shown in the bottom dataset.

\section{CONCLUSIONS}

A finite element model of a residual stress-free reference specimen has been successfully created for a hole in plate specimen. This simulated dataset has been shown to have very little variation from the experimentally derived reference data, an expected amount of experimental noise is present in the subtracted dataset. Further work will investigate using the artificial reference data in place of the experimental data to try and replicate results found by Robinson et al in identifying areas affected by residual stress.

\section{REFERENCES}

[1] Dulieu-Barton, J.M., Thermoelastic Stress Analysis [Optical Methods for Solid Mechanics: A Full-Field Approach], P. Rastogi and E. Hack, Editors. Wiley-VCH Verlag GmbH \& Co. KGaA. (2012).

[2] Steinzig, M., and Takahashi, T., "Residual stress measurement using the hole drilling method and laser speckle interferometry," Experimental Techniques 27(6), 59-63 (2006).

[3] Turski, T., and Edwards, L., "Residual stress measurement of a 315L stainless steel bead-on-plate specimen utilising the contour method," International Journal of Pressure Vessels and Piping 86, 126-131 (2009).

[4] Withers, P.J., and Bhadeshia, H.K.D.H., "Overview: Residual Stress Part 1 - Measurement Techniques," Materials Science and Technology 17, 355-365 (2001).

[5] Quinn, S., Dulieu-Barton, J.M., Eaton-Evans, J.M., Fruehmann, R.K., and Tatum, P.J., "Thermoelastic assessment of plastic deformation," Journal of Strain Analysis 43, 451-468 (2008).

[6] Vishay Precision Group, "Measurement of Residual Stresses by the Hole-Drilling Strain Gage Method," Micro Measurements [Vishay PG], (2010).

[7] Bray, D.E., Tang, W., and Grewal, D., "Ultrasonic stress evaluation in a turbine/compressor rotor," Journal of Testing and Evaluation 25, 503-509 (1997).

[8] Belgen, M.H., "Structural stress measurements with an infrared radiometer," ISA transactions 6, 49-53 (1967).

[9] Wong, A.K., Sparrow, J.G., and Dunn, S., "On the revised theory of the thermoelastic effect," Journal of Physics and Chemistry of Solids 49, 395-400 (1988).

[10] Wong, A.K., Dunn, S.A., and Sparrow, J.G., "Residual Stress measurement by means of the thermoelastic effect," Nature 332, 613-615 (1988).

[11] Dunn, S.A., Lombardo, D., and Sparrow, J.G., "The mean stress effect in metallic alloys and composites," SPIE Stress and Vibration: Recent Developments in Industrial Measurement and Analysis 1084, 129-142 (1989).

[12] Quinn, S., Dulieu-Barton, J.M., and Langlands, J.M., "Progress in thermoelastic residual stress measurement," Strain 40, 127-133 (2004). 
[13] Robinson, A.F., Dulieu-Barton, J.M., Quinn, S., and Burguete, R.L., "The Potential for Assessing Residual Stress Using Thermoelastic Stress Analysis: A Study of Cold Expanded Holes,” Experimental Mechanics 53, 299-317 (2013). 